\title{
EQUIVARIANT VECTOR BUNDLES OVER CLASSIFYING SPACES FOR PROPER ACTIONS
}

\author{
DIETER DEGRIJSE AND IAN J. LEARY
}

\begin{abstract}
Let $G$ be an infinite discrete group and let $\underline{E} G$ be a classifying space for proper actions of $G$. Every $G$-equivariant vector bundle over $\underline{E} G$ gives rise to a compatible collection of representations of the finite subgroups of $G$. We give the first examples of groups $G$ with a cocompact classifying space for proper actions $\underline{E} G$ admitting a compatible collection of representations of the finite subgroups of $G$ that does not come from a $G$-equivariant (virtual) vector bundle over $\underline{E} G$. This implies that the Atiyah-Hirzeburch spectral sequence computing the $G$-equivariant topological K-theory of $\underline{E} G$ has non-zero differentials. On the other hand, we show that for right angled Coxeter groups this spectral sequence always collapses at the second page and compute the K-theory of the classifying space of a right angled Coxeter group.
\end{abstract}

\section{INTRODUCTION}

Let $G$ be an infinite discrete group and $\mathcal{F}$ be the family of finite subgroups of $G$. Recall that the orbit category $\mathcal{O}_{\mathcal{F}} G$ is a category whose objects are the transitive $G$-sets $G / H$, for all $H \in \mathcal{F}$, and whose morphism are all $G$-equivariant maps between the objects. A classifying space for proper actions of $G$, denoted by $\underline{E} G$, is a proper $G$-CW-complex such that the fixed point set $\underline{E} G^{H}$ is contractible for every $H \in \mathcal{F}$. The space $\underline{E} G$ is said to be cocompact if the orbit space $G \backslash \underline{E} G=\underline{B} G$ is compact. Many interesting classes of groups $G$ have cocompact models for $\underline{E} G$, for example cocompact lattices in Lie groups, mapping class groups of surfaces, $\operatorname{Out}\left(F_{n}\right), \mathrm{CAT}(0)$-groups and word-hyperbolic groups. We refer the reader to [9] for more examples and details.

Now assume $G$ is an infinite discrete group admitting a cocompact classifying space for proper actions $\underline{E} G$. If

$$
\xi: E \rightarrow \underline{E} G
$$

is a $G$-equivariant complex vector bundle over $\underline{E} G$ (see Definition 2.3) and $x$ is a point of $\underline{E} G$, then the fiber $\xi^{-1}(x)$ is a complex representation of the finite isotropy group $G_{x}$. The connectivity of the fixed point sets of $\underline{E} G$ ensures that these representations are compatible (see Definition 2.1) with one another as $x$ and hence $G_{x}$ varies. Therefore, every $G$-equivariant complex vector bundle over $\underline{E} G$ gives rise to a compatible collection of complex representations of the finite subgroups of $G$, and hence to an element of

$$
\lim _{G / H \in \mathcal{O}_{\mathcal{F}} G} R(H) \text {. }
$$

Date: May 19, 2016.

The first author was supported by the Danish National Research Foundation through the Centre for Symmetry and Deformation (DNRF92). 
Here, $\lim _{G / H \in \mathcal{O}_{\mathcal{F}} G} R(H)$ is the limit over the orbit category $\mathcal{O}_{\mathcal{F}} G$ of the contravariant representation ring functor

$$
R(-): \mathcal{O}_{\mathcal{F}} G \rightarrow \mathrm{Ab} \quad G / H \mapsto R(H) .
$$

Denoting the Grothendieck group of the abelian monoid of isomorphism classes of complex $G$-vector bundles over $\underline{E} G$ by $\mathrm{K}_{G}^{0}(\underline{E} G)$, one obtains a map

$$
\varepsilon_{G}: \mathrm{K}_{G}^{0}(\underline{E} G) \rightarrow \lim _{G / H \in \mathcal{O}_{\mathcal{F}} G} R(H)
$$

that maps a formal difference of (isomorphism classes) vector bundles (i.e. a virtual vector bundle) to a formal difference of (isomorphism classes) of compatible collections of representations of the finite subgroups of $G$. We say a compatible collection of represesentations of the finite subgroups of $G$ can be realized as a (virtual) $G$-equivariant vector bundle over $\underline{E} G$ if there exists a (virtual) $G$-equivariant vector bundle over $\underline{E} G$ that maps to this collection un$\operatorname{der} \varepsilon_{G}$. One can also look at the corresponding situation for real (orthogonal) vector bundles and real (orthogonal) representations and obtain the map

$$
\varepsilon_{G}: \mathrm{KO}_{G}^{0}(\underline{E} G) \rightarrow \lim _{G / H \in \mathcal{O}_{\mathcal{F}} G} R O(H) .
$$

The maps $\varepsilon_{G}$ are equal to the edge homomorphisms of certain Atiyah-Hirzebruch spectral sequences converging to $\mathrm{K}_{G}^{*}(\underline{E} G)$ and $\mathrm{KO}_{G}^{*}(\underline{E} G)$ (see (1) and (2)). Lück and Oliver proved that (see Proposition 2.5) the map $\varepsilon_{G}$ (real or complex) is rationally surjective, meaning that a high enough multiple of every element in the target of $\varepsilon_{G}$ is contained in the image of $\varepsilon_{G}$. In the last paragraph of [12, p. 596] Lück and Oliver ask for an example of a group $G$ admitting a cocompact classifying space for proper actions $\underline{E} G$ such that $\varepsilon_{G}$ is not surjective. In Section 3 of this paper we give the first example of such a group in the complex case. In Section 4 we give the first example of such a group in the real case. We also construct examples of groups $G$ admitting a cocompact $\underline{E} G$ with the following weaker property: $G$ admits a compatible collection of representations for its finite subgroups that cannot be realized as a $G$-vector bundle over $\underline{E} G$. However, for these examples we cannot exclude the possibility that there exists a virtual vector bundle that maps to this collection of representations under $\varepsilon_{G}$. On the other hand, these examples are more explicit and lower dimensional.

In the final section we show that for a right angled Coxeter group $W$, every compatible collection of representations of the finite subgroups of $W$ can be realized as a $W$-equivariant vector bundle over $\underline{E} W$, so that the map

$$
\varepsilon_{W}: \mathrm{K}_{W}^{0}(\underline{E} W) \rightarrow \lim _{W / H \in \mathcal{O}_{\mathcal{F}} W} R(H) .
$$

is always surjective. Morever, we show that this map is actually an isomorphism and that (see Theorem 2.4)

$$
\mathrm{K}_{W}^{1}(\underline{E} W)=0 .
$$

Using a version of the Atiyah-Segal completion theorem for infinite discrete groups proven by Lück and Oliver, we use these results to compute the complex K-theory of $B W$, the classifying space of $W$ (see Corollary 5.6).

\section{ACKNOWLEDGEMENT}

We thank the referee, whose comments on an earlier version of this article were extremely helpful. 


\section{G-VECTOR BUNDLES AND ISOTROPy REPRESENTATIONS}

Let $G$ be a discrete group and let $\Gamma$ be a Lie group. Let $\mathcal{S}$ be a family of finite subgroups of $G$, i.e. any collection of finite subgroups of $G$ that is closed under conjugation and passing to subgroups. The orbit category $\mathcal{O}_{\mathcal{S}} G$ is a category whose objects are the transitive $G$-sets $G / H$, for all $H \in \mathcal{S}$, and whose morphism are all $G$-equivariant maps between the objects.

Definition 2.1. [12, p. 590] Let $X$ be a $G$-CW-complex. A $(G, \Gamma)$-bundle over $X$ is a $\Gamma$ principal bundle $p: E \rightarrow X$, where $E$ is a left $G$-space such that $p$ is $G$-equivariant and such that the left $G$-action and the right $\Gamma$-action on $E$ commute. We denote the set of isomorphism classes of $(G, \Gamma)$-bundles over $X$ by $\operatorname{Bdl}_{(G, \Gamma)}(X)$. For $H \in \mathcal{F}$, let

$$
\operatorname{Rep}_{\Gamma}(H)=\operatorname{Hom}(H, \Gamma) / \operatorname{Inn}(\Gamma) .
$$

One can consider $\operatorname{Rep}_{\Gamma}(-)$ as a contravariant functor from $\mathcal{O}_{\mathcal{S}} G$ to Sets. An element of the limit

$$
A=\left(\left[\alpha_{H}\right]\right)_{H \in \mathcal{S}} \in \lim _{G / H \in \mathcal{O}_{\mathcal{S}} G} \operatorname{Rep}_{\Gamma}(H)
$$

is a called an $\mathcal{S}$-compatible collection of $\Gamma$-representations. Given such an element $A$, let $\mathcal{S}_{A}$ be the family of subgroups of $G \times \Gamma$ consisting of conjugates of the subgroups of the form

$$
\left\{\left(h, \alpha_{H}(h)\right) \mid h \in H\right\}
$$

for all $H \in \mathcal{S}$ and let $E_{\mathcal{S}}(G, A)$ be the universal $G \times \Gamma$-CW-complex for the family $\mathcal{S}_{A}$.

Lemma 2.2. [12, Lemma 2.4] For every $\mathcal{S}$-compatible collection of $\Gamma$-representations $A=$ $\left(\left[\alpha_{H}\right]\right)_{H \in \mathcal{S}}$ there exists a $G$-CW-complex $B_{\mathcal{S}}(G, A)$ with isotropy in $\mathcal{S}$ satisfying the following properties.

- The quotient map

$$
\pi: E_{\mathcal{S}}(G, A) \rightarrow \Gamma \backslash E_{\mathcal{S}}(G, A)=B_{\mathcal{S}}(G, A)
$$

is a $(G, \Gamma)$-bundle over the $G$ - $C W$-complex $B_{\mathcal{S}}(G, A)$.

- The $(G, \Gamma)$-bundle $\pi: E_{\mathcal{S}}(G, A) \rightarrow B_{\mathcal{S}}(G, A)$ is universal in the sense that for every $G$-CW-complex $X$ with isotropy in $\mathcal{S}$ there is an isomorphism

$$
\left[X, \mathcal{B}_{\mathcal{S}}(G, A)\right]_{G} \cong \operatorname{Bld}_{(G, \Gamma)}(X)
$$

given by pulling back the univeral bundle $\pi$ along a $G$-map $X \rightarrow \mathcal{B}_{\mathcal{S}}(G, A)$.

- For every $S \in \mathcal{S}$, the fixed point set $B_{\mathcal{F}}(G, A)^{H}$ is homotopy equivalent to $B C_{\Gamma}\left(\alpha_{H}\right)$, the classifying space of the centralizer of the image of $\alpha_{H}$ in $\Gamma$.

If $\Gamma=\mathrm{U}(n)(\Gamma=\mathrm{O}(n))$ and $\mathcal{S}=\mathcal{F}$, the family of all finite subgroups of $G$, then $\operatorname{Rep}_{\Gamma}(H)$ is the set of isomorphism classes of $n$-dimensional complex (real) representations of $H$. In this case, an element of the limit

$$
A=\left(\left[\alpha_{H}\right]\right)_{H \in \mathcal{F}} \in \lim _{G / H \in \mathcal{O}_{\mathcal{F}} G} \operatorname{Rep}_{\Gamma}(H)
$$

is a called is called a compatible collection of complex (real) $n$-dimensional representations of the finite subgroups of $G$. For $H \in \mathcal{F}$, let $R(H)(R O(H))$ be the complex (real) representation ring of $H$, i.e. the Grothendieck group of the abelian cancellative monoid of isomorphism classes of finite dimensional complex (real) representations of $H$. Note that $\operatorname{Rep}_{\mathrm{U}(\mathrm{n})}(H)$ is 
naturally a subset of $R(H)$ and $\operatorname{Rep}_{\mathrm{O}(\mathrm{n})}(H)$ is naturally a subset of $R O(H)$. One can consider $R(-)$ as a functor from $\mathcal{O}_{\mathcal{F}} G$ to $\mathrm{Ab}$. An element of the inverse limit

$$
\alpha=\left(\left[\alpha_{H}\right]\right)_{H \in \mathcal{F}} \in \lim _{G / H \in \mathcal{O}_{\mathcal{F}} G} R(H)
$$

is a called a compatible collection of complex virtual representations of the finite subgroups of $G$. One has a natural embedding

$$
\lim _{G / H \in \mathcal{O}_{\mathcal{F}} G} \operatorname{Rep}_{\mathrm{U}(n)}(H) \subset \lim _{G / H \in \mathcal{O}_{\mathcal{F}} G} R(H) .
$$

The analogous statements for $\mathrm{O}(n, \mathbb{R})$ and $R O$ also hold.

Now let $X$ be a proper cocompact $G$-CW-complex, i.e. $X$ has finite isotropy and the orbit space $G \backslash X$ has a finite number of cells, such that for every $H \in \mathcal{F}$, the fixed point set $X^{H}$ is non-empty and connected.

Definition 2.3 ([18]). A complex (real) $G$-vector bundle over $X$ is a complex (real) vector bundle $\pi: E \rightarrow X$ such that $\pi$ is $G$-equivariant and each $g \in G$ acts on $E$ and $X$ via a bundle isomorphism. An isomorphism of $G$-vector bundles over $X$ is just an isomorphism of vector bundles that is $G$-equivariant. The set of isomorphims classes of complex (real) $G$-vector bundles over $X$ will be denoted by $\operatorname{Bdl}_{G}(X)\left(\operatorname{OBdl}_{G}(X)\right)$. For every $x \in X$, the fiber $\pi^{-1}(x)$ is denoted by $E_{x}$. We refer the reader to [12, Section 1] and [20, Section I.9] for elementary properties of $G$-vector bundles over proper (cocompact) $G$-CW complexes.

Theorem 2.4. [12, Th. 3.2 and 3.15] There exists a 2-periodic (8-periodic) equivariant cohomology theory $\mathrm{K}_{G}^{*}(X, A)\left(\mathrm{KO}_{G}^{*}(X, A)\right)$ on the category of proper $G$-CW-pairs such that when $X$ is cocompact, $\mathrm{K}_{G}^{0}(X)\left(\mathrm{KO}_{G}^{0}(X)\right)$ is the Grothendieck group of the abelian monoid of isomorphism classes of complex (real) $G$-vector bundles over $X$. In particular, for every $H \in \mathcal{F}, \mathrm{K}_{G}^{0}(G / H)\left(\mathrm{KO}_{G}^{0}(G / H)\right)$ is canonically isomorphic to $R(H)(R O(H))$.

As usual (see [13, Section 6] and [5, Th. 4.7]), the skeletal filtration of $X$ induces AtiyahHirzebruch spectral sequences

$$
E_{2}^{p, q}=\mathrm{H}_{G}^{p}\left(X, \mathrm{~K}_{G}^{q}(G /-)\right) \Longrightarrow \mathrm{K}_{G}^{p+q}(X) .
$$

and

$$
E_{2}^{p, q}=\mathrm{H}_{G}^{p}\left(X, \mathrm{KO}_{G}^{q}(G /-)\right) \Longrightarrow \mathrm{KO}_{G}^{p+q}(X)
$$

where $\mathrm{H}_{G}^{p}(X,-)$ denotes Bredon cohomology of $X$ (see [2]).

Proposition 2.5. [13, Prop 5.8] If $X$ is a cocompact $G$-CW complex then the spectral sequences (1) and (2) above rationally collapse, meaning that the images of all differentials in these spectral sequences consist of torsion elements.

By our assumptions on $X$, the zeroth Bredon cohomology group $\mathrm{H}_{G}^{0}(X, R(-)$ ) (resp. $\mathrm{H}_{G}^{0}(X, R O(H))$ ), equals the limit of the functor $R(-)$ (resp. $R O(-)$ ), over the orbit category $\mathcal{O}_{\mathcal{F}} G$. Consider the edge homomorphisms

$$
\varepsilon_{G}: \mathrm{K}_{G}^{0}(X) \rightarrow \mathrm{H}_{G}^{0}(X, R(-))
$$

and

$$
\varepsilon_{G}: \mathrm{KO}_{G}^{0}(X) \rightarrow \mathrm{H}_{G}^{0}(X, R O(-))
$$


of the spectral sequences (1) and (2). If $[\pi]$ is the isomorphism class of an $n$-dimensional complex $G$-vector bundle $\pi: E \rightarrow X$, then $\varepsilon_{G}([\pi])$ equals

$$
\left(\left[E_{e_{H}}\right]\right)_{H \in \mathcal{F}} \in \lim _{G / H \in \mathcal{O}_{\mathcal{F}} G} \operatorname{Rep}_{U(n)}(H) \subset \mathrm{H}_{G}^{0}(X, R(-))
$$

where $\left[E_{e_{H}}\right]$ denotes the isomorphism class in $R(H)$ of the $H$-representation $E_{e_{H}}$. The corresponding statement for real $G$-vector bundles also holds. Note that it follows from Proposition 2.5 that a suitable multiple of every compatible collection of (virtual) real or complex representations of the finite subgroups of $G$ is contained in the image of the edge homomorphism $\varepsilon_{G}$.

Recall that the classifying space for proper actions $\underline{E} G$ is a terminal object in the homotopy category of proper $G$-CW complexes (e.g. [9, Th. 1.9]). Hence, if $X$ is any proper cocompact $G$-CW complex such that $X^{H}$ is non-empty and connected for each $H \in \mathcal{F}$, then there exists a $G$-map $X \rightarrow \underline{E} G$ that is unique up to $G$-homotopy and induces commutative diagrams

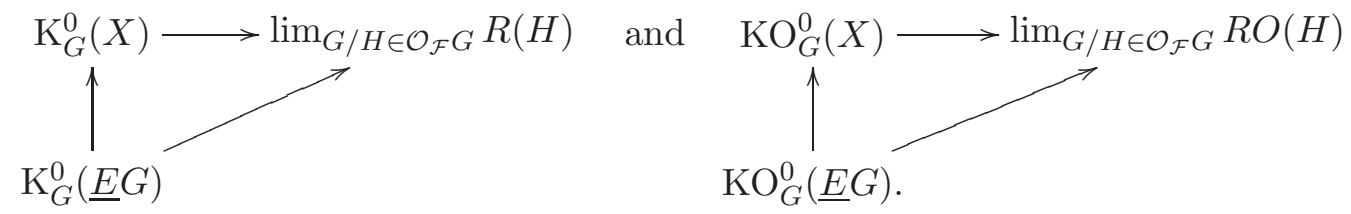

Hence, if a compatible collection $\alpha$ of virtual representations can be realized as a virtual $G$-vector bundle over $\underline{E} G$, it can also be realized as a virtual $G$-vector bundle over $X$.

\section{Complex vector Bundles}

The purpose of this section is to construct a group $G$ with a cocompact classifying space for proper actions $\underline{E} G$ admitting a compatible collection of complex representations of the finite subgroups of $G$ that cannot be realized as $G$-equivariant virtual complex vector bundle over $\underline{E} G$, i.e. so that the edge homomorphism

$$
\varepsilon_{G}: \mathrm{K}_{G}^{0}(\underline{E} G) \rightarrow \lim _{G / H \in \mathcal{O}_{\mathcal{F}} G} R(H)
$$

is not surjective.

Let $F=C_{4} \rtimes C_{2}$ be the dihedral group of order 8 where $\sigma$ is generator for $C_{4}$ and $\varepsilon$ is a generator of $C_{2}$. Let $H=\left\langle\sigma^{2}\right\rangle$ be the center of $F$, which has order two and denote the $n$-skeleton of the universal $F / H$-space $X=E(F / H)$ by $X^{n}$. We let $F$ act on $X$ and $X^{n}$ via the projection onto $F / H$. Consider the complex 1-dimensional representation

$$
\lambda: H=\left\langle\sigma^{2}\right\rangle \rightarrow \mathrm{U}(1)=S^{1}: \sigma^{2} \mapsto-1 .
$$

Lemma 3.1. The isomorphism class $[\lambda]$ is contained in $R(H)^{F / H}$. For $k \in \mathbb{Z}$, the multiple $k[\lambda]$ is contained in the image of the restriction map res: $R(F) \rightarrow R(H)$ if and only if $k$ is even.

Proof. Since $H$ is the center of $F$ it follows that the conjugation action of $F / H$ on $R(H)$ is trivial, hence $[\lambda] \in R(H)^{F / H}=R(H)$. One easily verifies that the representation

$$
\tau: F \rightarrow U(2)
$$


defined by

$$
\tau(\sigma)=\left(\begin{array}{cc}
0 & i \\
i & 0
\end{array}\right) \text { and } \tau(\varepsilon)=\left(\begin{array}{cc}
-1 & 0 \\
0 & 1
\end{array}\right)
$$

satisifies $\operatorname{res}([\tau])=2[\lambda]$. Hence, $k[\lambda]$ is contained in the image of res for every even $k \in \mathbb{Z}$. Note that, as a free abelian group, $R(H)$ is generated by $[\lambda]$ and the isomorphism class of the 1-dimensional complex trivial representation [tr] (e.g. see [19]). Now suppose $k$ is odd and there exists an element $[\mu]-[\rho] \in R(F)$ such that $\operatorname{res}([\mu]-[\rho])=k[\lambda]$. There are integers $l, m, n$ and such that $\operatorname{res}([\mu])=l[\operatorname{tr}]+m[\lambda], \operatorname{res}([\rho])=l[\operatorname{tr}]+n[\lambda]$ and $m-n=k$. By changing the representative of $[\mu]$, we may also assume that

$$
\mu: F \rightarrow \mathrm{U}(l+m)
$$

where $\mu(\sigma)$ is a diagonal matrix. Since $\mu\left(\sigma^{2}\right)$ has an $m$-dimensional eigenspace with eigenvalues -1 and an $l$-dimensional eigenspace with eigenvalue 1 , it follows that $\mu(\sigma)$ has an $s$-dimensional eigenspace with eigenvalue $i$ and a $t$-dimensional eigenspace with eigenvalue $-i$ such that $s+t=m$. Morever, $\mu\left(\sigma^{3}\right)$ has an $s$-dimensional eigenspace with eigenvalue $-i$ and a $t$-dimensional eigenspace with eigenvalue $i$. Since $\sigma$ and $\sigma^{3}$ are conjugate in $F$, it follows that $s=t$ proving that $m$ is even. A similiar argument shows that $n$ is also even. But this contradicts the fact that $k=m-n$ is odd. Hence, there does not exist an element $[\mu]-[\rho] \in R(F)$ such that $\operatorname{res}([\mu]-[\rho])=k[\lambda]$, if $k$ is odd.

The following lemma uses the notation introduced above and will be cited in the next section.

Lemma 3.2. Every F-equivariant complex line bundle over $X^{3}$ is isomorphic to the pullback of an $F$-equivariant complex line bundle over $E(F / H)$ along the inclusion $i: X^{3} \rightarrow E(F / H)$.

Proof. Let $\mathcal{S}$ be the family of subgroups of $F$ containing only $H$ and the trivial subgroup. Note that isomorphism classes of $F$-equivariant complex line bundles correspond to isomorphism classes of $\left(F, S^{1}=\mathrm{U}(1)\right)$-bundles. Let $\pi: E \rightarrow X^{3}$ be an $F$-equivariant complex line bundle over and let $\left[\alpha_{H}: H \rightarrow \mathrm{U}(1)=S^{1}\right]$ be the isomorphism class in $\operatorname{Rep}_{S^{1}}(H)$ of the $H$ representation induced on the fibers of $\pi$. If we set $\alpha_{\{e\}}:\{e\} \rightarrow S^{1}$, then $A=\left(\left[\alpha_{K}\right]\right)_{K \in \mathcal{S}} \in$ $\lim _{K \in \mathcal{S}} \operatorname{Rep}_{S^{1}}(K)$. It follows from Lemma 2.2 for $\Gamma=S^{1}$, that in order to show that $\pi$ is the pullback of an $F$-equivariant complex line bundle over $E(F / H)$ along the inclusion $i: X^{3} \rightarrow E(F / H)$, it suffices to show that every $F$-map from $X^{3}$ to $B_{\mathcal{S}}(F, A)$ can be extended to an $F$-map from $E(F / H)$ to $B_{\mathcal{S}}(F, A)$. Here $B_{\mathcal{S}}(F, A)$ is homotopy equivalent to $B S^{1}=\mathbb{C} P^{\infty}$ for all $K \in \mathcal{S}$, again by Lemma 2.2. It follows from Bredon's equivariant obstruction theory (see [2, Section II.1],[15, Th. I.5.1]) that the potential obstructions for extending such a map lie in the relative Bredon cohomology groups $\mathrm{H}_{F}^{n+1}\left(E(F / H), X^{3} ; \pi_{n}\left(\mathrm{~B}_{\mathcal{S}}(F, A)^{-}\right)\right)$ for $n \geq 3$. Since $\pi_{n}\left(\mathbb{C} P^{\infty}\right)$ is zero unless $n=2$, the lemma is proven.

The idea for the following lemma is contained in [12, p 596].

Lemma 3.3. There exists an $n \geq 1$ such that $[\lambda]$ is not contained in the image of the edge homomorphism

$$
\mathrm{K}_{F}^{0}\left(X^{n}\right) \rightarrow R(H)^{F / H} .
$$

Proof. By [7, Theorem 5.1] for $X=\{*\}, \mathcal{F}=\{e, H\}$ and $E \mathcal{F}=E(F / H)$, there are maps

$$
\alpha_{n}: R(F) / I^{n} \rightarrow \mathrm{K}_{F}^{0}\left(X^{n}\right)
$$


that induce a map of inverse systems from $\left\{R(F) / I^{n}\right\}_{n \geq 0}$ to $\left\{\mathrm{K}_{F}^{0}\left(X^{n}\right)\right\}_{n \geq 0}$ that induces an isomorphism of pro-rings. Here $I$ is the kernel of the restriction map $R(F) \rightarrow R(H)$. This implies that for sufficiently large $n \geq 1$ there exists a map $\beta_{1}: \mathrm{K}_{F}^{0}\left(X^{n}\right) \rightarrow R(F) / I$ making the following diagram commute

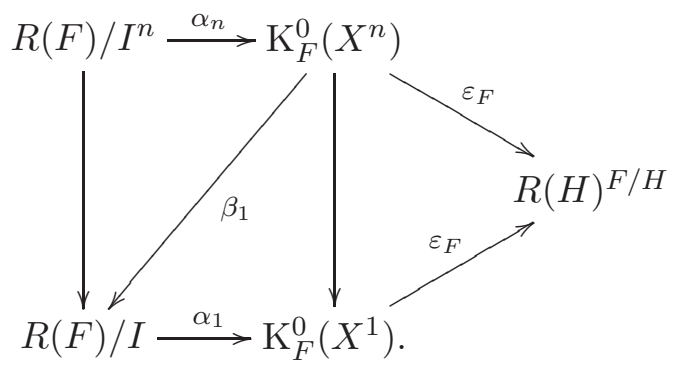

This shows that the image of the restriction map

$$
R(F) \rightarrow R(H)^{F / H}
$$

coincides with the image of the edge homomorphism

$$
\mathrm{K}_{F}^{0}\left(X^{n}\right) \rightarrow R(H)^{F / H} .
$$

Since $[\lambda]$ does not lie in the image of $\mathrm{R}(F) \rightarrow \mathrm{R}(H)^{F / H}$ by Lemma 3.1, the lemma follows.

Let $n \geq 3$. By [8, Th. A \& Th. 8.3] there exists a compact $n$-dimensional locally CAT(0)cubical complex $T_{X^{n}}$ equipped with a free cellular $F / H$-action and an $F / H$-equivariant map $t_{X^{n}}: T_{X^{n}} \rightarrow X^{n}$ that induces an isomorphism

$$
\mathcal{H}_{F}^{*}\left(X^{n}\right) \stackrel{\cong}{\rightrightarrows} \mathcal{H}_{F}^{*}\left(T_{X^{n}}\right)
$$

for any equivariant cohomology theory $\mathcal{H}_{?}^{*}(\cdot)$ (e.g. see $[11$, section 1$\left.]\right)$. (We remark that $[8$, Th. 8.3] is stated for equivariant homology theories, but the analogous statement holds for equivariant cohomology theories by essentially the same proof.) The action of $F$ on $T_{X^{n}}$ in the above is via the projection $F \rightarrow F / H$. Now let $Y^{n}$ be the universal cover of $T_{X^{n}}$ and let $\Gamma_{n}$ be the group of self-homeomorphisms of $Y^{n}$ that lift the action of $F / H$ on $T_{X^{n}}$. Since $F / H$ acts freely on $T_{X^{n}}, \Gamma_{n}$ acts freely on $Y^{n}$. We conclude that $Y^{n}$ is an $n$-dimensional CAT(0)cubical complex on which $\Gamma_{n}$ acts freely, cocompactly and cellularly. Since $Y_{n}$ is contractible, this implies that $\Gamma_{n}$ is torsion-free. By construction there is a surjection $\Gamma_{n} \rightarrow F / H$ whose kernel $N_{n}$ is the torsion-free group of deck transformation of the covering $Y^{n} \rightarrow T_{X^{n}}$. Now define the group $G_{n}$ to be the pullback of $\pi_{n}: \Gamma_{n} \rightarrow F / H$ along $F \rightarrow F / H$. Then $G_{n}$ acts on $Y^{n}$ via the quotient $\operatorname{map} G_{n} \rightarrow G_{n} / H=\Gamma_{n}$ and fits into the short exact sequence

$$
1 \rightarrow N_{n} \rightarrow G_{n} \stackrel{p_{n}}{\longrightarrow} F \rightarrow 1 .
$$

Note that the only non-trivial finite subgroup of $G_{n}$ is $H \cong C_{2}$ and that since $N_{n}$ acts freely on $Y^{n}$, the $G_{n}$-equivariant quotient map $Y^{n} \rightarrow N_{n} \backslash Y^{n}=T_{X^{n}}$ induces an isomorphism ([12, Lemma 3.5])

$$
\mathrm{K}_{F}^{*}\left(T_{X^{n}}\right) \stackrel{\cong}{\rightrightarrows} \mathrm{K}_{G_{n}}^{*}\left(Y^{n}\right) .
$$


Applying (3) and (4) to the compostion $Y^{n} \rightarrow T_{X^{n}} \rightarrow X^{n}$ and the equivariant cohomology theories $\mathrm{K}_{?}^{*}(\cdot)$ and $\mathrm{H}_{?}^{*}(\cdot, R(-))$ with $*=0$, we obtain a commutative diagram

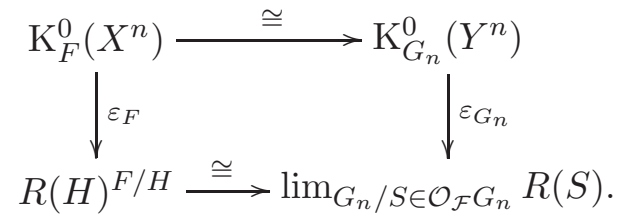

The fact that this diagram commutes can be seen as follows. Using equivariant cellular approximation, we may assume that the map $X^{n} \rightarrow Y^{n}$ is cellular. By considering the inclusion of zero-skeleta in $n$-skeleta, naturality yields a commutative diagram

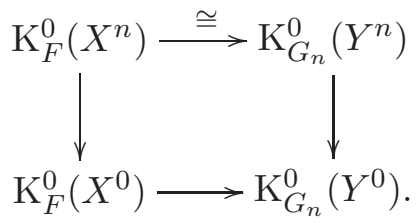

The edge homomorphism $\varepsilon_{F}: \mathrm{K}_{F}^{0}\left(X^{n}\right) \rightarrow R(H)^{F / H} \subseteq \mathrm{K}_{F}^{0}\left(X^{0}\right)$ coincides by construction with $\mathrm{K}_{F}^{0}\left(X^{n}\right) \rightarrow \mathrm{K}_{F}^{0}\left(X^{0}\right)$ once we restrict the codomain, and similarly for $\varepsilon_{G_{n}}$. Therefore, commutativity follows.

Since we proved in Lemma 3.3 that, for $n$ large enough, the isomorphism class of $\lambda$ does not lie in the image of the edge homomorphism

$$
\mathrm{K}_{F}^{0}\left(X^{n}\right) \rightarrow R(H)^{F / H}
$$

it follows from the commutative diagram above that the compatible system of representations

$$
\left(\lambda \circ p_{n \mid S}\right)_{S \in \mathcal{F}} \in \lim _{G_{n} / S \in \mathcal{O}_{\mathcal{F}} G_{n}} R(S)=\mathrm{H}_{\mathcal{F}}^{0}\left(G_{n}, R(-)\right) .
$$

does not lie in the image of the edge homomorphism

$$
\varepsilon_{G_{n}}: \mathrm{K}_{G_{n}}^{0}\left(Y^{n}\right) \rightarrow \lim _{G_{n} / S \in \mathcal{O}_{\mathcal{F}} G_{n}} R(S) .
$$

Recall from [3] that non-empty CAT(0)-cube complexes are contractible and that the fixed point set for a finite group action on a CAT(0)-cube complex is contractible. Since $G_{n}$ acts cellularly properly and cocompactly on the CAT(0)-cube complex $Y_{n}$, we deduce that $Y_{n}$ is a cocompact model for $\underline{E} G_{n}$. To summarize, we have constructed a group $G=G_{n}$ with a cocompact classifying space for proper actions $\underline{E} G$ admitting a compatible collection of complex representations of the finite subgroups of $G$ that cannot be realized as $G$-equivariant virtual complex vector bundle over $\underline{E} G$.

We remark that Wolfgang Lück has shown us another quite different way to find a finite group $F$ and an $F$-CW-complex $X$ that satisfy Lemma 3.3; any such pair could be used to construct a group with similar properties to the group $G=G_{n}$.

\section{Real Vector Bundles}

One could apply the techniques of the previous section in the real setting to obtain a group $G$ with cocompact classifying space for proper actions $\underline{E} G$ so that the edge homomorphism

$$
\varepsilon_{G}: \mathrm{KO}_{G}^{0}(\underline{E} G) \rightarrow \lim _{G / H \in \mathcal{O}_{\mathcal{F}} G} R O(H)
$$


is not surjective. Here one would need the real version of [7, Theorem 5.1], which also holds as explained in the paragraph below [7, Theorem 5.1].

Instead we give an explicit description of a group $G$ that admits $\mathbb{R}^{2}$ as a cocompact model for $\underline{E} G$ and admits a compatible collection of real representations of its finite subgroups that cannot be realized as a real $G$-vector bundle over $\mathbb{R}^{2}$.

We start by describing a related group $\Gamma$ that is a 2-dimensional crystallographic group, or wallpaper group; this group is known as $p 2 g g$, but we will describe it explicitly. Endow $\mathbb{R}^{2}$ with the CW-structure coming from the standard tesselation by unit squares with vertices at $\mathbb{Z}^{2}$, and let $\Gamma$ be the group of automorphisms of this CW-structure that preserves the pattern shown in Figure 1. The stabilizer of a 2-cell is clearly trivial, and so the 2-cells form a single free $\Gamma$-orbit. There are two orbits of 1 -cells, the vertical and horizontal edges, and again each orbit is free. There are two orbits of 0-cells, and the stabilizer of a 0-cell is cyclic of order two, generated by the rotation of order two fixing the point. Since the stabilizer of each cell acts trivially on that cell, the given $\mathrm{CW}$-structure makes $\mathbb{R}^{2}$ into a $\Gamma$-CW-complex.

The translation subgroup $T$ of $\Gamma$ has index four, and consists of the elements $(x, y) \mapsto$ $(x+2 m, y+2 n)$. The orientation-preserving subgroup $N$ of $\Gamma$ has index two, and consists of $T$ together with the rotations through $\pi$ about some point of $\mathbb{Z}^{2}$, which are of the form $(x, y) \mapsto$ $(2 m-x, 2 n-y)$. Finally the elements of $\Gamma-N$ are the glide reflections whose axes bisect the sides of the 2-cells: $(x, y) \mapsto(2 m+1-x, 2 n+1+y)$ and $(x, y) \mapsto(2 m+1+x, 2 n+1-y)$. The quotients $T \backslash \mathbb{R}^{2}, N \backslash \mathbb{R}^{2}$ and $\Gamma \backslash \mathbb{R}^{2}$ are respectively a torus consisting of four squares, an $S^{2}$ obtained by identifying the boundaries of two squares, and a copy of $\mathbb{R} P^{2}$ obtained by identifying the edges of a square in pairs. The fact that $\Gamma-N$ contains no torsion elements is reflected in the fact that $\Gamma / N$ acts freely on the sphere $N \backslash \mathbb{R}^{2}$.

Now let $F$ be a copy of $C_{4}$ and let $H \cong C_{2}$ be the index two subgroup of $F$. The group $G$ is defined as the pullback of the two maps $\Gamma \rightarrow \Gamma / N \cong C_{2}$ and $F \rightarrow F / H \cong C_{2}$. By construction the group $G$ admits $\mathbb{R}^{2}$ as a cocompact model for $\underline{E} G$, and fits into a short exact sequence

$$
1 \rightarrow N \rightarrow G \stackrel{p}{\rightarrow} F \rightarrow 1
$$

such that every finite subgroup of $G$ maps onto a subgroup of $H$ under $p$.

Now let

$$
\lambda: H \rightarrow O(1, \mathbb{R})=C_{2}
$$

be the 1-dimensional real sign representation of $H$, i.e. $\lambda$ is the identity map. The isomorphism class $[\lambda]$ is clearly contained in $R O(H)^{F / H}$, since $F$ is abelian.

Lemma 4.1. The isomorphism class $k[\lambda]$ is contained in the image of the restriction map

$$
R O(F) \rightarrow R O(H)^{F / H} .
$$

if and only if $k$ is even.

Proof. Recall that the irreducible real representations of $C_{4}$ are up to isomorphism the onedimensional trivial representation representation, the one dimensional sign representation of $F / H=C_{2}$ and one 2-dimensional faithful representation in which the elements of order four act as rotations by $\pm \frac{\pi}{2}$. The restriction of the first two of the representations to $H$ gives the trivial one dimensional representation of $H$, while the restriction to $H$ of the third is $\lambda \oplus \lambda$. We therefore conclude that the image of $R O(F) \rightarrow R O(H)^{F / H}$ consists of element of the form $2 n[\lambda]+m[\operatorname{tr}]$, where $\operatorname{tr}$ is the trivial one dimensional representation of $H$ and $n, m \in \mathbb{Z}$. 


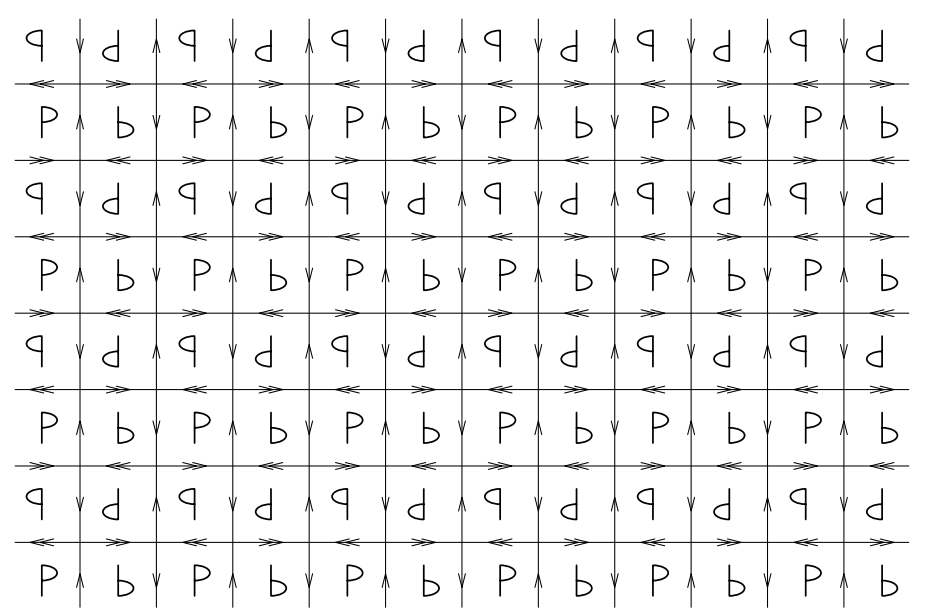

Figure 1. A wallpaper pattern for $\Gamma=p 2 g g$

This shows that $k[\lambda]$ is contained in the image of the restriction map $R O(F) \rightarrow R O(H)^{F / H}$ if and only if $k$ is even.

Lemma 4.2. Let $F$ act on the infinite dimensional sphere $S^{\infty}$ by first projecting onto $\mathrm{F} / \mathrm{H}=\mathrm{C}_{2}$ and then acting via the antipodal map. View $S^{2}$ as the 2-skeleton of $S^{\infty}$. Every F-equivariant orthogonal real line bundle over $S^{2}$ is isomorphic to the pullback of an $F$-equivariant orthogonal real line bundle over $S^{\infty}$ along the inclusion $S^{2} \rightarrow S^{\infty}$.

Proof. Let $\mathcal{S}$ be the family of subgroups of $F$ containing $H$ and the trivial subgroup. Note that isomorphism classes of $F$-equivariant orthogonal real line bundles correspond to isomorphism classes of $\left(F, C_{2}\right)$-bundles. Now let $\xi$ be an $\left(F, C_{2}\right)$-bundle over $S^{2}$ with fibers $A=\left(\xi_{S}\right) \in$ $\lim _{S \in \mathcal{S}} \operatorname{Rep}_{C_{2}}(S)$. By Lemma 2.2, it suffices to show that every $F$-map $f: S^{2} \rightarrow \mathrm{B}_{\mathcal{S}}(F, A)$ can be extended to an $F$-map $\tilde{f}: S^{\infty} \rightarrow \mathrm{B}_{\mathcal{S}}(F, A)$. Again by Lemma $2.2, \mathrm{~B}_{\mathcal{S}}(F, A)^{S} \cong B C_{2}=$ $\mathbb{R} P^{\infty}$ for all $S \in \mathcal{S}$. It follows from Bredon's equivariant obstruction theory (see [2, Section II.1],[15, Th. I.5.1]) that the potential obstructions for extending such a map lie in the relative Bredon cohomology groups $\mathrm{H}_{F}^{n+1}\left(S^{\infty}, S^{2} ; \pi_{n}\left(\mathrm{~B}_{\mathcal{S}}(F, A)^{-}\right)\right)$for $n \geq 2$. Since $\pi_{n}\left(\mathbb{R} P^{\infty}\right)$ is zero unless $n=1$, the lemma is proven.

Lemma 4.3. Let $F$ act on $S^{2}$ by first projecting onto $F / H=C_{2}$ and then acting via the antipodal map. There does not exist a real F-vector bundle $\xi: E \rightarrow S^{2}$ such that the representation of $H$ on the fibers of $\xi$ is isomorphic to $\lambda$.

Proof. Consider the infinite dimensional sphere $S^{\infty}$ as a the universal $C_{2}$-space $E C_{2}$, where $C_{2}$ acts via the antipodal map and let $F$ act on $S^{\infty}$ via first projection onto $F / H=C_{2}$ and then acting via $C_{2}$. Now assume that there exists a real $F$-vector bundle $\xi: E \rightarrow S^{2}$ such that the representation of $H$ on the fibers of $\xi$ is isomorphic to $\lambda$. By Lemma 4.2 there exists a real $F$-vector bundle $\xi: E \rightarrow S^{\infty}$ such that the representation of $H$ on the fibers of $\xi$ is isomorphic to $\lambda$. By pulling back this bundle along the inclusion $S^{n} \rightarrow S^{\infty}$, there also exists a real $F$-vector bundle $\xi: E \rightarrow S^{n}$ such that the representation of $H$ on the fibers of $\xi$ is isomorphic to $\lambda$, for every $n \geq 2$. 
By the real version of $[7$, Theorem 5.1] (see comments below [7, Theorem 5.1] ), there are maps

$$
\alpha_{n}: R O(F) / I^{n} \rightarrow \mathrm{KO}_{F}^{0}\left(S^{n}\right)
$$

that induce a map of inverse systems from $\left\{R O(F) / I^{n}\right\}_{n \geq 0}$ to $\left\{\operatorname{KO}_{F}^{0}\left(S^{n}\right)\right\}_{n \geq 0}$ that in turn induces an isomorphism of pro-rings. Here $I$ is the kernel of the restriction map $R O(F) \rightarrow$ $R O(H)$. This implies that for sufficiently large $n \geq 1$ there exists a map $\beta_{1}: \mathrm{KO}_{F}^{0}\left(S^{n}\right) \rightarrow$ $R(F) / I$ making the following diagram commute

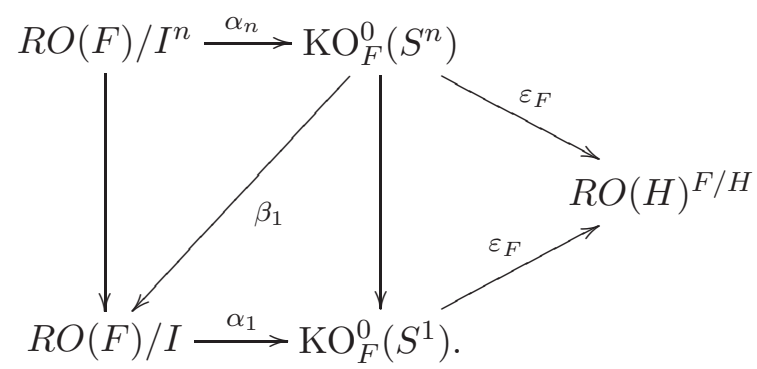

This shows that the image of the restriction map

$$
R O(F) \rightarrow R O(H)^{F / H}
$$

coincides with the image of the edge homomorphism

$$
K O_{F}^{0}\left(S^{n}\right) \rightarrow R O(H)^{F / H},
$$

implying that the $H$-representations coming from the fibers of any real $F$-vector bundle over $S^{n}$ can be extended to virtual $F$-representations. However, since $\lambda$ does not lie in the image of $\mathrm{RO}(F) \rightarrow \mathrm{RO}(H)$ by Lemma 4.1 we arrive at a contradiction and conclude that there does not exist a real $F$-vector bundle $\xi: E \rightarrow S^{2}$ such that the representation of $H$ on the fibers of $\xi$ is isomorphic to $\lambda$.

Consider the projection $p: G \rightarrow F$ and the compatible system of real orthogonal representations

$$
\left(\left[\lambda \circ p_{\mid S}\right]\right)_{S \in \mathcal{F}} \in \lim _{G / S \in \mathcal{O}_{\mathcal{F}} G} R O(S)=\mathrm{H}_{G}^{0}(\underline{E} G, R O(-)),
$$

and assume that there exists a real $G$-vector bundle $\xi: E \rightarrow \mathbb{R}^{2}$ that realizes it. Since the kernel of $p: G \rightarrow F$ is $N$, it follows from the lemma below and our observations above that $N \backslash \xi: N \backslash E \rightarrow N \backslash X$ is an $F$-vector bundle over $S^{2}$, where $F$ acts on $S^{2}$ via projection onto $F / H=C_{2}$, followed by the antipodal map. Morever, the representation of $H$ on the fibers of $N \backslash \xi$ is by construction exactly $\lambda$. This however contradicts Lemma 4.3, so we conclude that there does not exist a real $G$-vector bundle $\xi: E \rightarrow \mathbb{R}^{2}$ that realizes the compatible system of real orthogonal representations $\left(\lambda \circ p_{\mid S}\right)_{S \in \mathcal{F} \text {. }}$

Lemma 4.4. Let $G$ be any discrete group with normal subgroup $N$ and let $X$ be a proper $G$-CW-complex. If $\xi: E \rightarrow X$ is a G-vector bundle over $X$ such that $N \cap G_{x}$ acts trivially on $\xi^{-1}(x)$ for every $x \in X$, then

$$
N \backslash \xi: N \backslash E \rightarrow N \backslash X
$$

is a $G / N$-vector bundle over $N \backslash X$. 
Proof. Denote the projection $G \rightarrow G / N=Q$ by $\pi$. Let us first consider the case where $\xi$ is trivial (trivial in the sense of [10, Section 6.1.]), i.e. assume $\xi$ is a pullback

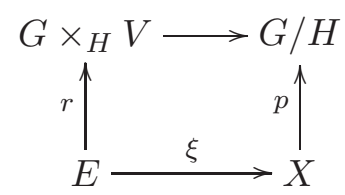

of the $G$-vector bundle $G \times_{H} V \rightarrow G / H$ along the $G$-map $p: X \rightarrow G / H$ where $H$ is some finite subgroup of $G$ and $V$ is a finite dimensional real $H$-representation such that $H \cap N$ acts trivially on $V$. Consider the pullback diagram

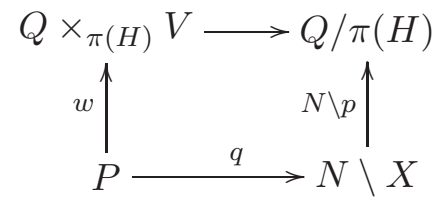

of the $Q$-vector bundle $Q \times_{\pi(H)} V \rightarrow Q / \pi(H)$ along the $Q$-map $N \backslash p: N \backslash X \rightarrow Q / \pi(H)$. We define the map

$$
\psi: N \backslash E \rightarrow P: \overline{(g, v, x)} \mapsto(\pi(g), v, \bar{x}) .
$$

It is easy to check that $\psi$ yields a well-defined morphism of $Q$-equivariant bundles over $N \backslash X$. Moreover, since $\psi$ is a fiberwise linear map of $Q$-vector bundles that is a fiberwise isomorphism, it follows that $\psi$ is a homeomorphism.

Now consider the general case. Let $\bar{x} \in N \backslash X$. Since $\xi: E \rightarrow X$ is locally trivial, $x \in X$ has an open $G$-neighourhood $U$ such that there is a $G$-map $p: U \rightarrow G / H$ where $H$ is finite subgroup of $G$ and $\xi_{\mid U}$ is (homeomorphic to) the pullback

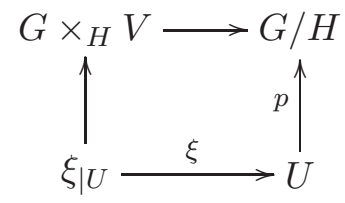

of the $G$-vector bundle $G \times_{H} V \rightarrow G / H$ along the $G$-map $p: U \rightarrow G / H$. By the above, the quotient diagram

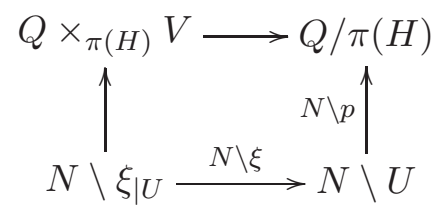

is a pullback diagram. Since $N \backslash U$ is an open $Q$-neighbourhood of $\bar{x}$, it follows that $N \backslash$ $\xi: N \backslash E \rightarrow N \backslash X$ is a $Q$-vector bundle.

We finish this section by explaining how a similar approach to the one above can be used to produce a group $G$ admitting a three dimensional cocompact model for $\underline{E} G$ that has a compatible system of one-dimensional complex representations that cannot be realized as a complex $G$-vector bundle over $\underline{E} G$. As in Section 3, let $F=C_{4} \rtimes C_{2}$ be the dihedral group of order 8 where $\sigma$ is a generator for $C_{4}$. Let $H=\left\langle\sigma^{2}\right\rangle$ be the center of $F$, which has order two 
and denote the 3 -skeleton of the universal $F / H$-space $X=E(F / H)$ by $X^{3}$. We let $F$ act on $X$ and $X^{3}$ via the projection onto $F / H$. Consider the complex 1-dimensional representation

$$
\lambda: H=\left\langle\sigma^{2}\right\rangle \rightarrow \mathrm{U}(1)=S^{1}: \sigma^{2} \mapsto-1 .
$$

By [8, Th. A \& Th. 8.3] there exists a compact 3-dimensional locally CAT(0)-cubical complex $T_{X^{3}}$ equipped with a free cellular $F / H$-action, an $F / H$-equivariant map $t_{X^{3}}: T_{X^{3}} \rightarrow X^{3}$ and an isometric cellular involution $\tau$ on $T_{X^{3}}$ that commutes with the $F / H$-action on $T_{X^{3}}$ and the map $t_{X^{3}}$ such the induced $F / H$-equivariant map

$$
\langle\tau\rangle \backslash T_{X^{3}} \rightarrow X^{3}
$$

is a homotopy equivalence. Note that $F / H$ acts freely on $\langle\tau\rangle \backslash T_{X^{3}}$ since it acts freely on $X^{3}$. Hence $T_{X^{3}}$ is also the 3 -skeleton of a universal $F / H$-space $Z$. So we may continue assuming that $Z=X$ and $\langle\tau\rangle \backslash T_{X^{3}}=X^{3}$.

Now let $Y$ be the univeral cover of $T_{X^{3}}$ and let $\Gamma$ be the group of self-homeomorphism of $Y$ that lifts the action of $F / H \oplus\langle\tau\rangle$ on $T_{X^{3}}$. Then $Y$ is a 3-dimensional CAT(0)-cubical complex on which $\Gamma$ acts properly, compactly and cellularly. By construction there is a surjection $\alpha: \Gamma \rightarrow F / H \oplus\langle\tau\rangle$ whose kernel $\operatorname{Ker}(\alpha)$ is the torsion-free group of deck transformations of $Y \rightarrow T_{X^{3}}$. Let $\pi$ denote the composition of $\alpha$ with the projection of $F / H \oplus\langle\tau\rangle$ onto $F / H$. Since $F / H$ acts freely on $T_{X^{3}}$ and every finite subgroup of $\Gamma$ must fix a point of $Y$ since $Y$ is $\mathrm{CAT}(0)$, it follows that every finite subgroup of $\Gamma$ is contained in the kernel of $\pi$, which we denote by $N$. Now define the group $G$ to be the pullback of $\pi: \Gamma \rightarrow F / H$ along $F \rightarrow F / H$. Then $G$ acts on $Y$ via the quotient map $G \rightarrow G / H=\Gamma$ that fits into the short exact sequence

$$
1 \rightarrow N \rightarrow G \stackrel{p}{\rightarrow} F \rightarrow 1 \text {. }
$$

such that $p$ maps all the finite subgroup of $G$ onto a finite subgroup of $H$ and $N \backslash Y=X^{3}$.

Let $\mathcal{F}$ be the family of finite subgroups of $G$, note that $Y$ is a three dimensional cocompact model for $\underline{E} G$ and suppose that there exists a $G$-vector $\xi: E \rightarrow Y$ whose fibers give rise to the compatible system of representations

$$
\left(\left[\lambda \circ p_{\mid S}\right]\right)_{S \in \mathcal{F}} \in \lim _{G / S \in \mathcal{O}_{\mathcal{F}} G} R(S) .
$$

Applying Lemma 4.4, we obtain an $F$-equivariant complex line bundle $N \backslash \xi: N \backslash E \rightarrow X$ such that the representation of $H$ on the fibers of $N \backslash \xi$ is isomorphic to $\lambda$. By Lemma 3.2, this bundle can be extended to an $F$-equivariant complex line bundle over $X=E(F / H)$. We now continue in a similar fashion as in the proof of Lemma 4.3 to conclude that $[\lambda]$ is contained in the image of the restriction map $R(F) \rightarrow R(H)^{F / H}$, which contradicts Lemma 3.1. We conclude that the bundle $\xi$ cannot exist.

\section{Right Angled Coxeter groups}

Let $\Gamma$ be a finite graph. We denote the vertex set of $\Gamma$ by $S=V(\Gamma)$ and the set edges of $\Gamma$ by $E(\Gamma) \subseteq V(\Gamma) \times V(\Gamma)$. The right angled Coxeter group determined by $\Gamma$ is the Coxeter group $W$ with presentation

$$
\left.W=\langle S| s^{2} \text { for all } s \in V(\Gamma) \text { and }(s t)^{2} \text { if }(s, t) \in E(\Gamma)\right\rangle .
$$

Note that $W$ fits into the short exact sequence

$$
1 \rightarrow N \rightarrow W \stackrel{p}{\rightarrow} F=\bigoplus_{s \in S} C_{2} \rightarrow 1
$$


where $p$ takes $s \in S$ to the generator of the $C_{2}$-factor corresponding to $s$. A subset $J \subseteq S$ is called spherical if the subgroup $W_{J}=\langle J\rangle$ is finite (and hence isomorphic to $\bigoplus_{s \in J} C_{2}$ ). The empty subset of $S$ is by definition spherical. We denote the poset of spherical subsets of $S$ orderded by inclusion by $\mathcal{S}$. If $J \in \mathcal{S}$, then $W_{J}$ is called a spherical subgroup of $W$, while a coset $w W_{J}$ is called spherical coset. We denote the poset of spherical cosets, ordered by inclusion, by $W \mathcal{S}$. Note that $W$ acts on $W \mathcal{S}$ by left multiplication, preserving the ordering. The Davis complex $\Sigma$ of $W$ is the geometric realization of $W \mathcal{S}$. One easily sees that $\Sigma$ is a proper cocompact $W$-CW-complex. Since $\Sigma$ admits a complete CAT(0)-metric such that $W$ acts by isometries, it follows that $\Sigma$ is a cocompact model for $\underline{E} W$ (see [4, Th. 12.1.1 \& Th. 12.3.4]). A consequence of this fact is that every finite subgroup of $W$ is subconjugate to some spherical subgroup of $W$. This implies that the group $N$ defined above is torsion-free. Since the quotient space $W \backslash \Sigma$ is homeomorphic to the geometric realization of the poset $\mathcal{S}$, which is contractible since it has a minimal element, another consequence is that the quotient $\underline{B} W=W \backslash \underline{E} W$ is contractible. We refer the reader to [4] for more details and information about these groups and the spaces on which they act.

Let $\mathcal{F}$ be the family of finite subgroups of $W$. Given an abelian group $A$, we denote by

$$
\underline{A}: \mathcal{O}_{\mathcal{F}} W \rightarrow \mathrm{Ab}
$$

the trivial functor that takes all objects to $A$ and all morphism to the identity map. One can verify that

$$
\mathrm{H}_{W}^{*}(\underline{E} W, \underline{A}) \cong \mathrm{H}^{*}(\underline{B} W, A) .
$$

Lemma 5.1. Let $A=\left(\left[p_{\mid H}\right]\right)_{H \in \mathcal{F}} \in \lim _{W / H \in \mathcal{O}_{\mathcal{F}} W} \operatorname{Rep}_{F}(H)$. For every $k \geq 0$, the contravariant functor

equals the trivial functor $\pi_{k}(B F)$.

$$
\mathcal{O}_{\mathcal{F}} W \rightarrow \mathrm{Ab}: W / H \mapsto \pi_{k}\left(B_{\mathcal{F}}(W, A)^{H}\right)
$$

Proof. Let $E F$ be a contractible $F-C W$-complex with free $F$-action and consider the product space $\underline{E} W \times E F$. This space becomes a $(W \times K)-C W$-complex by letting $(w, f) \in W \times F$ act on $(x, y) \in \underline{E} W \times E F$ as

$$
(w, f) \cdot(x, y)=(w \cdot x, p(w) f \cdot y) .
$$

One checks that with this action $\underline{E} W \times E F$ is a model for $E_{\mathcal{F}}(W, A)$, i.e. $(\underline{E} W \times E F)^{K}$ is contractible when $K \in \mathcal{F}_{A}$ and empty otherwise. By definition, it follows that $\underline{E} W \times B F$ is a model $B_{\mathcal{F}}(W, A)$, where $W$ acts on trivially on the second coordinate. Since $\underline{E} W^{H}$ is contractible for every $H \in \mathcal{F}$, the lemma follows easily.

Let $\Gamma$ be either the orthogonal group $\mathrm{O}(n, \mathbb{R})$ or the unitary group $\mathbf{U}(n)$.

Lemma 5.2. Every element of

$$
\lim _{W / H \in \mathcal{O}_{\mathcal{F}} W} \operatorname{Rep}_{\Gamma}(H)
$$

is of the form $\left(\left[\lambda \circ p_{\mid H}\right]\right)_{H \in \mathcal{F}}$ for some group homomorphism $\lambda: F \rightarrow \Gamma$.

Proof. Every finite subgroup $H$ of $W$ is isomorphic to a finite direct sum of $C_{2}$ 's. Since every element of order 2 in $\Gamma$ is conjugate in $\Gamma$ to a diagonal matrix with \pm 1 on the diagonal and commuting matrices can be simultaneously diagonalized (e.g. see [6, Th. 1.3.12]), it follows that the image of every homomorphism $H \rightarrow \Gamma$ is conjugate to a finite subgroup of $\Gamma$ consisting 
of diagonal matrices. Hence, every element of $\lim _{W / H \in \mathcal{O}_{\mathcal{F}} W} \operatorname{Rep}_{\Gamma}(H)$ is of the form $\left(\left[\alpha_{H}\right]\right)_{H \in \mathcal{F}}$ where $\alpha_{H}: H \rightarrow \Gamma$ is a homomorphism whose image lands in the finite abelian subgroup of $\Gamma$ consisting of diagonal matrices. Since every finite subgroup of $W$ is subconjugate to a spherical subgroup $W_{J}$, the compatibility of the representations tells us that $\left(\left[\alpha_{H}\right]\right)_{H \in \mathcal{F}}$ is completely determined by the homomorphisms $\alpha_{\langle s\rangle}:\langle s\rangle \rightarrow \Gamma$, for $s \in S$. Since the images of the $\alpha_{\langle s\rangle}$ are diagonal, they commute. Therefore, one can define the homomorphism

$$
\lambda: F=\bigoplus_{s \in S} C_{2} \rightarrow \Gamma:\left(\sigma_{s}\right)_{s \in S} \mapsto \sum_{s \in S} \alpha_{\langle s\rangle}\left(\sigma_{s}\right) .
$$

The compatibility of the representations implies that

$$
\left(\left[\lambda \circ p_{\mid H}\right]\right)_{H \in \mathcal{F}}=\left(\left[\alpha_{H}\right]\right)_{H \in \mathcal{F}},
$$

proving the lemma.

The following theorem applies to both complex and real representations and vector bundles.

Theorem 5.3. Let $W$ be a right angled Coxeter group. Every compatible collection of representations of the finite subgroups of $W$ can be realized as a $W$-equivariant vector bundle over the Davis complex $\Sigma=\underline{E} W$.

Proof. Consider $A=\left(\left[p_{\mid H}\right]\right)_{H \in \mathcal{F}} \in \lim _{W / H \in \mathcal{O}_{\mathcal{F}} W} \operatorname{Rep}_{F}(H)$. It follows from Lemma 2.2 that the existence of a $(W, A)$-bundle over $\Sigma$ follows from the existence a $W$-map $\Sigma \rightarrow B_{\mathcal{F}}(W, A)$. Since by Lemma 5.1 , the contravariant functor

$$
\pi_{k}\left(B_{\mathcal{F}}(W, A)^{-}\right): \mathcal{O}_{\mathcal{F}}(W) \rightarrow \mathrm{Ab}: W / H \mapsto \pi_{k}\left(B_{\mathcal{F}}(W, A)^{H}\right)
$$

equals the trivial functor $\pi_{k}(B F)$ for all $k \geq 0$, it follows from (5) and the contractibility of $\underline{B} W$ that the Bredon cohomology groups

$$
\mathrm{H}_{W}^{k+1}\left(\Sigma, \pi_{k}\left(B_{\mathcal{F}}(W, A)^{-}\right)\right)
$$

are zero for all $k \geq 0$. Since there certainly exists a $W$-map from the 0-skeleton of $\Sigma$ to $B_{\mathcal{F}}(W, A)$, it follows from Bredon's equivariant obstruction theory that there exists a $W$-map $\Sigma \rightarrow B_{\mathcal{F}}(W, A)$.

Now consider a compatible collection of representations of the finite subgroups of $W$. By Lemma 5.2, this collection is of the form

$$
\left(\left[\lambda \circ p_{\mid H}\right]\right)_{H \in \mathcal{F}} \in \lim _{W / H \in \mathcal{O}_{\mathcal{F}} W} \operatorname{Rep}_{\Gamma}(H)
$$

for some group homomorphism $\lambda: F \rightarrow \Gamma$. Letting $A=\left(\left[p_{\mid H}\right]\right)_{H \in \mathcal{F}}$, it follows from the above that there exists a $(W, A)$-bundle $\xi: E \rightarrow \Sigma$. If $\Gamma=\mathrm{O}(n, \mathbb{R})$ then

$$
\xi: E \times_{F} \mathbb{R}^{n} \rightarrow \Sigma
$$

is a real $W$-vector bundle over $\Sigma$ that realizes $\left(\left[\lambda \circ p_{\mid H}\right]\right)_{H \in \mathcal{F}}$, and if $\Gamma=U(n)$ then

$$
\xi: E \times{ }_{F} \mathbb{C}^{n} \rightarrow \Sigma
$$

is a complex $W$-vector bundle over $\Sigma$ that realizes $\left(\left[\lambda \circ p_{\mid H}\right]\right)_{H \in \mathcal{F}}$. Here $F$ acts on $\mathbb{R}^{n}$ or $\mathbb{C}^{n}$ via the map $\lambda$.

Lemma 5.4. If $W$ is a right angled Coxeter group, then $\mathrm{H}_{W}^{n}(\Sigma, R(-))=0$ for all $n>0$, and $\mathrm{H}_{W}^{0}(\Sigma, R(-))$ is free abelian of rank equal to the number of spherical subgroups of $W$. 
Proof. This is proven in much the same way as the corresponding result for homology in [17]. In more detail, one uses the cubical structure on $\Sigma$, in which there is one orbit of $n$-cubes with stabilizer isomorphic to $\left(C_{2}\right)^{n}$ for each $n$-tuple of commuting elements of $S$. (For each $n \geq 0$, for each spherical subgroup $W_{J} \cong\left(C_{2}\right)^{n}$ and for each $w \in W$, the subposet consisting of all special cosets contained in $w W_{J}$ is order isomorphic to the poset of faces of an $n$-cube. Furthermore this isomorphism is equivariant for the stabilizer subgroup $w W_{J} w^{-1} \cong\left(C_{2}\right)^{n}$, acting on the $n$-cube as the group generated by reflections in its coordinate planes. The realizations of these subposets are the cubes that make up the cubical structure on $\Sigma$. For more details concerning the cubical structure on $\Sigma$ see [4, Ch. 1.1-1.2 or Ch. 7].) Since the stabilizer of a cube of strictly positive dimension acts non-trivially on the cube, this cubical structure is not a $W$-CW-structure on $\Sigma$. However, its barycentric subdivision is a simplicial complex naturally isomorphic to the realization of the poset $W \mathcal{S}$ as described in the introduction to this section.

Let $\Sigma^{n}$ denote the $n$-skeleton of $\Sigma$ with the cubical structure. Firstly, $\Sigma^{0}$ consists of a single free $W$-orbit of vertices, so $\mathrm{H}_{W}^{*}\left(\Sigma^{0} ; R(-)\right)$ is isomorphic to the ordinary cohomology of a point; since $W$ acts freely the calculation reduces to an equivariant cohomology calculation for the trivial group action.

Let $I=[-1,1]$ be an interval, with $C_{2}$ acting by $x \mapsto-x$ (i.e., swapping the ends of the interval). Note that $I$ is equivariantly isomorphic to the Davis complex for the Coxeter group $C_{2}$. Let $\partial I$ denote the two end points $\{-1,1\}$. Make $I$ into a $C_{2}-\mathrm{CW}$-complex, for example by taking three 0 -cells in two orbits at the points $-1,0$ and 1 , and one free orbit of 1-cells consisting of the two intervals $[-1,0]$ and $[0,1]$. The cellular $C_{2}$-Bredon cochain complex for the pair $(I, \partial I)$ with coefficients in $R(-)$ is a cochain complex of free abelian groups in which the degree zero term has rank two, the degree one term has rank one, and all other terms are trivial. A direct computation with this cochain complex shows that $H_{C_{2}}^{m}(I, \partial I ; R(-))$ is isomorphic to $\mathbb{Z}$ for $m=0$ and is zero for $m>0$.

Next consider $I^{n}$ with $C_{2}^{n}$ acting as the direct product of $n$ copies of the above action of $C_{2}$ on $I$. This is the Davis complex for the Coxeter group $C_{2}^{n}$. Since the representation ring of a direct product of finite groups is naturally identified with the tensor product of the representation rings $\left[19\right.$, Ch. 3.2], the $C_{2}^{n}$-Bredon cochain complex for the pair $\left(I^{n}, \partial I^{n}\right)$ with coefficients in $R(-)$ is naturally isomorphic to the tensor product of $n$ copies of the $C_{2^{-}}$ Bredon cochain complex for $(I, \partial I)$ with coefficients in $R(-)$. (If one wants to think about this cochain complex geometrically, it arises from the $\left(C_{2}\right)^{n}$-CW-structure on $I^{n}$ in which the cells are the direct products of the cells arising in the $C_{2}$-CW-structure on $I$.) Since these cochain complexes consist of finitely generated free abelian groups, there is a Künneth formula as described in for example [16, Thrm 60.3]. Since $H_{C_{2}}^{*}(I, \partial I ; R(-))$ is free abelian the Künneth formula implies that

$$
H_{C_{2}^{n}}^{*}\left(I^{n}, \partial I^{n}, R(-)\right) \cong \bigotimes_{i=1}^{n} H_{C_{2}}^{*}(I, \partial I ; R(-))
$$

It follows that for each $n, H_{C_{2}^{n}}^{m}\left(I^{n}, \partial I^{n} ; R(-)\right)$ is isomorphic to $\mathbb{Z}$ for $m=0$ and is zero for $m>0$.

From these computations, it follows easily that $H_{W}^{m}\left(\Sigma^{n}, \Sigma^{n-1} ; R(-)\right)$ is zero for $m>0$ and is isomorphic to a direct sum of copies of $\mathbb{Z}$ indexed by the $W$-orbits of $n$-cubes in $\Sigma$. By induction on $n$ one sees that $H_{W}^{m}\left(\Sigma^{n} ; R(-)\right)$ is zero for $m>0$ and isomorphic to a direct sum of copies of $\mathbb{Z}$ indexed by the $W$-orbits of cubes of dimension at most $n$ for $m=0$. The 
claimed result follows, since the $W$-orbits of cubes in $\Sigma$ are in bijective correspondence with the spherical subgroups of $W$.

Before stating our theorem concerning $\mathrm{K}_{W}^{*}(\underline{E} W)$, we make some remarks concerning the representation ring of a direct sum of copies of the cyclic group $C_{2}$, indexed by a (finite) set $S$. For any finite group $G$, the collection of all isomorphism types of 1-dimensional complex representations of $G$ is an abelian group, with product given by taking the tensor product of representations. Furthermore, this group is naturally isomorphic to the group $\operatorname{Hom}(G, \mathrm{U}(1))$. In the case when $G$ is abelian, every irreducible representation of $G$ is 1-dimensional, and so $\operatorname{Hom}(G, \mathrm{U}(1))$ forms a basis for the additive group of the representation ring. In this way the representation ring $R(G)$ is naturally isomorphic to the integral group algebra of the group $\operatorname{Hom}(G, \mathrm{U}(1))$. In the case when $G=\bigoplus_{s \in S} C_{2}$ is a direct sum of copies of $C_{2}$ indexed by $S$, we may view $G$ as a vector space over the field of two elements, in which case $\operatorname{Hom}(G, \mathrm{U}(1))$ may be identified with the dual space. For $s \in S$, let $s^{*}$ denote the 1-dimensional representation of $G$ with the properties that $s^{*}(s)=-1$ and $s^{*}(t)=1$ for $t \in S-\{s\}$. let $S^{*}$ denote the set of these representations: $S^{*}:=\left\{s^{*} \mid s \in S\right\}$. In terms of vector spaces over the field of two elements, $S^{*} \subseteq \operatorname{Hom}(G, \mathrm{U}(1))$ is the dual basis to the set $S \subseteq G$. The set $S^{*}$ generates the representation ring of $G$, giving rise to the following presentation:

$$
R(G)=\mathbb{Z}\left[S^{*}\right] /\left(s^{* 2}-1 \mid s \in S\right),
$$

in which the monomials $s_{1}^{*} s_{2}^{*} \cdots s_{k}^{*}$ for all subsets $\left\{s_{1}, \ldots, s_{k}\right\} \subseteq S$ correspond to the irreducible representations.

Suppose now that $J$ is a subset of $S$. The inclusion $J \subseteq S$ identifies $H=\bigoplus_{s \in J} C_{2}$ with a subgroup of $G=\bigoplus_{s \in J} C_{2}$. The induced map $R(G) \rightarrow R(H)$ of representation rings is described easily in terms of the above ring presentation: for $s \in J, s^{*} \in R(G)$ restricts to $s^{*} \in R(H)$, while for $s \notin J, s^{*} \in R(G)$ restricts to $1 \in R(H)$.

Now suppose that $\Gamma$ is a graph with vertex set $V(\Gamma)=S$, and let $W$ be the right angled Coxeter group associated to $\Gamma$. The abelianization of $W$ is naturally identified with $G=$ $\bigoplus_{s \in S} C_{2}$. There is a unique equivariant map $\alpha: \underline{E} W \rightarrow *$, from the $W$-space $\underline{E} W$ to a point *, viewed as a $G$-space with trivial action. If $J$ is a spherical subset of $S$ then $W_{J}=\bigoplus_{s \in J} C_{2}$ maps isomorphically to the corresponding subgroup of $G=\bigoplus_{s \in S} C_{2}$. If $x \in \underline{E} W$ is a $0-$ cell fixed by $W_{J}=\bigoplus_{s \in J} C_{2}$, then $\alpha(x)=*$, and this map is $W_{J}$-equivariant. The induced map $\alpha^{*}: \mathrm{K}_{G}^{*}(*) \rightarrow \mathrm{K}_{W}^{*}(\underline{E} W)$, and the composite map $\mathrm{K}_{G}^{*}(*) \rightarrow \mathrm{K}_{W_{J}}^{*}(\{x\})$ will be used in the statement and proof of our theorem. If we identify $R(G)$ with $\mathrm{K}_{G}^{0}(*)$ and $R\left(W_{J}\right)$ with $\mathrm{K}_{W_{J}}^{0}(\{x\})$, then the composite is identified with the restriction map.

Theorem 5.5. Let $W$ be the right angled Coxeter group determined by a finite graph $\Gamma$, with vertex set $S$, and let $G=\bigoplus_{s \in S}$ be the abelianization of $W$. The map $\alpha^{*}: \mathrm{K}_{G}^{*}(*) \rightarrow \mathrm{K}_{W}^{*}(\underline{E} W)$ is surjective in each degree. In particular, $\mathrm{K}_{W}^{1}(\underline{E} W)=0$ and there is a ring isomorphism

$$
\mathrm{K}_{W}^{0}(\underline{E} W) \cong \mathbb{Z}\left[S^{*}\right] /\left(s^{* 2}-1, s^{*} t^{*}-s^{*}-t^{*}+1 \mid s \in S=V(\Gamma),(s, t) \notin E(\Gamma)\right) .
$$

It follows that $\mathrm{K}_{W}^{0}(\underline{E} W) \cong \mathbb{Z}^{d}$ as an abelian group, where $d$ is the number of spherical subgroups of $W$.

Proof. Consider the Atiyah-Hirzebruch spectral sequence (1)

$$
E_{2}^{p, q}=\mathrm{H}_{W}^{p}\left(\underline{E} W, \mathrm{~K}_{W}^{q}(W /-)\right) \Longrightarrow \mathrm{K}_{W}^{p+q}(\underline{E} W)
$$


where $\mathrm{K}_{W}^{q}(W /-)=R(-)$ if $q$ is even and $\mathrm{K}_{W}^{q}(W /-)=0$ if $q$ is odd (see [12, Th. 3.2]). In the lemma above, we proved that $\mathrm{H}_{W}^{k}(\Sigma, R(-))=0$ for $k>0$. It therefore follows that

$$
\mathrm{K}_{W}^{n}(\underline{E} W)=\left\{\begin{array}{cc}
\mathrm{H}_{W}^{0}(\underline{E} W, R(-))=\lim _{W / H \in \mathcal{O}_{\mathcal{F} W}} R(H) & \text { if } n=0 \\
0 & \text { if } n=1 .
\end{array}\right.
$$

Let $I$ be the ideal

$$
\left(s^{* 2}-1, s^{*} t^{*}-s^{*}-t^{*}+1 \mid s \in S,(s, t) \notin E(\Gamma)\right)
$$

in the polynomial ring $\mathbb{Z}\left[S^{*}\right]$. Note that as an abelian group $\mathbb{Z}\left[S^{*}\right] / I$ is free, with basis elements the commuting products $s_{1}^{*} \ldots s_{k}^{*}$, for all $J=\left\{s_{1} \ldots, s_{k}\right\} \in \mathcal{S}$ (The case $J=\emptyset$ corresponds to the unit of $\mathbb{Z}[V(\Gamma)] / I)$. This shows that

$$
\mathbb{Z}\left[S^{*}\right] / I \cong \mathbb{Z}^{d}
$$

as an abelian group, where $d$ is the number of spherical subgroups of $W$.

We claim there is an isomorphism of rings

$$
\lim _{W / H \in \mathcal{O}_{\mathcal{F}} W} R(H) \cong \mathbb{Z}\left[S^{*}\right] / I .
$$

Since every finite subgroup of $W$ is subconjugate to a spherical subgroup of $W$, it follows that

$$
\lim _{W / H \in \mathcal{O}_{\mathcal{F}} W} R(H) \cong \lim _{J \in \mathcal{S}} R\left(W_{J}\right)
$$

as rings. By the remarks in the paragraph preceeding the statement of the theorem, there are ring isomorphisms

$$
R\left(W_{J}\right)=\mathbb{Z}\left[J^{*}\right] /\left(s^{* 2}-1 \mid s \in J\right), \quad R(G)=\mathbb{Z}\left[S^{*}\right] /\left(s^{* 2}-1 \mid s \in S\right),
$$

which are natural for inclusions $J \subseteq J^{\prime} \subseteq S$. From this it follows that the natural ring homomorphism

$$
\rho: R(G) \rightarrow \lim _{W / H \in \mathcal{O}_{\mathcal{F} W}} R(H)
$$

is surjective, and that $\lim _{W / H \in \mathcal{O}_{\mathcal{F} W}} R(H)$ is isomorphic to the ring described in the statement; in particular its additive group is free abelian of the same rank as $\mathrm{K}_{W}^{0}(\underline{E} W)$. Since $\rho$ factors through $\mathrm{K}_{W}^{0}(\underline{E} W)$, the claimed isomorphism follows.

Before stating our corollary concerning $\mathrm{K}^{*}(B W)$, we recall some facts from [1] concerning $\mathrm{K}^{*}(B G)$, where as above $G=\bigoplus_{s \in S} C_{2}$. For any finite group $H$, Atiyah showed that $\mathrm{K}^{i}(B H)=0$ for $i$ odd, and that $\mathrm{K}^{2 i}(B H)$ is naturally isomorphic to the completion of the representation ring $R(H)$ at its augmentation ideal. To discuss the case of $G$, it is convenient to take new generators for $R(G)$; replace the irreducible representation $s^{*}$ by the degree zero virtual representation $\bar{s}=s^{*}-1$. With respect to these generators one obtains the presentation

$$
R(G)=\mathbb{Z}[\bar{S}] /(\bar{s}(\bar{s}+2) \mid s \in S),
$$

where $\bar{S}=\{\bar{s} \mid s \in S\}$. If $H=\bigoplus_{s \in J} C_{2}$, then of course there is a similar description of $R(H)$, which is natural for the inclusion $J \subseteq S$. Note that if $s \notin J$, then the image of $\bar{s}$ under the restriction map $R(G) \rightarrow R(H)$ is zero.

Completing $R(G)$, as described above, with respect to its augmentation ideal gives rise to the following presentation for the ring $\mathrm{K}^{0}(B G)$ :

$$
\mathrm{K}^{0}(B G)=\mathbb{Z}[[\bar{S}]] /(\bar{s}(\bar{s}+2) \mid s \in S),
$$


which is natural for the inclusion $J \subseteq S$, and so also describes the induced map $\mathrm{K}^{0}(B G) \rightarrow$ $\mathrm{K}^{0}(B H)$. The additive group of this ring is the direct sum of one copy of $\mathbb{Z}$, generated by 1 , and for each non-empty subset $J \subseteq S$, one copy of the 2-adic integers, $\mathbb{Z}_{2}$, consisting of the set of power series in the element $\prod_{s \in J} \bar{s}$ with zero constant term.

Corollary 5.6. Let $W$ be the right angled Coxeter group determined by a finite graph $\Gamma$ with vertex set $S=V(\Gamma)$, and let $G=\bigoplus_{s \in S} C_{2}$ be the abelianization of $W$. The induced map $\mathrm{K}^{*}(B G) \rightarrow \mathrm{K}^{*}(B W)$ is surjective in each degree. In particular $\mathrm{K}^{1}(B W)=0$ and there is a ring isomorphism

$$
\mathrm{K}^{0}(B W) \cong \mathbb{Z}[[\bar{S}]] /(\bar{s}(\bar{s}+2), \bar{s} \bar{t} \mid s \in S,(s, t) \notin E(\Gamma)) .
$$

Here, $\mathbb{Z}[[\bar{S}]]$ is the formal power series ring with $\mathbb{Z}$ coefficients in the variables $\bar{S}=\{\bar{s} \mid s \in S\}$.

Proof. The version of the Atiyah-Segal completion theorem that is proven for infinite discrete groups admitting a cocompact model for the classifying space for proper actions in $[12$, Theorem 4.4.(b)] implies that

$$
\mathrm{K}^{n}(B W)=\mathrm{K}_{W}^{n}(\underline{E} W)_{\hat{J}}
$$

where the ideal $J$ is the kernel of the augmentation map $\mathrm{K}_{W}^{n}(\underline{E} W) \rightarrow \mathbb{Z}$ that maps vector bundles to their dimension. Changing variables in the above theorem to $\bar{s}=s^{*}-1$, we see that $\mathrm{K}^{i}(B W)=0$ for $i$ odd and that $\mathrm{K}^{0}(B W)$ is the completion of the ring

$$
\mathbb{Z}[\bar{S}] /(\bar{s}(\bar{s}+2), \bar{s} \bar{t} \mid s \in S,(s, t) \notin E(\Gamma))
$$

with respect to the ideal generated by the set $\bar{S}=\{\bar{s} \mid s \in S\}$. This completion is the ring described in the statement.

There is an alternative proof of Corollary 5.6 that does not use Theorem 5.5 or results from [12]. Instead one uses a description of $W$ as a free product with amalgamation. If the graph $\Gamma$ is not a complete graph, then there is an expression $\Gamma=\Gamma_{1} \cup \Gamma_{2}, \Gamma_{3}=\Gamma_{1} \cap \Gamma_{2}$, in which each $\Gamma_{i}$ is a full subgraph of $\Gamma$ and has fewer vertices than $\Gamma$. This gives an expression for $W$ as a free product with amalgamation $W=W_{1} *_{W_{3}} W_{2}$. From this one obtains a Mayer-Vietoris sequence that can be used to compute $\mathrm{K}^{*}(B W)$. To establish Corollary 5.6, one shows by induction on $|S|$ that $\mathrm{K}^{*}(B W)$ is as described and that for each $J \subseteq S$, the map $\mathrm{K}^{*}(B W) \rightarrow \mathrm{K}^{*}\left(B W_{J}\right)$ is a split surjection.

\section{REFERENCES}

[1] Atiyah, M. F., Characters and cohomology of finite groups, Inst. Hautes Études Sci. Publ. Math. 9 (1961) 23-64.

[2] Bredon, G. E., Equivariant cohomology theories, Lecture Notes in Mathematics 34, Springer (1967).

[3] Bridson, M. R. and Haefliger, A., Metric spaces of non-positive curvature Springer Verlag Vol. 319 (1999).

[4] Davis, M. W., The geometry and topology of Coxeter groups, Princeton University Press, vol. 32 (2008).

[5] Davis, J. F. and Lück, W., Spaces over a category and assembly maps in isomorphism conjectures in $K$ and $L$ - theory, $K$-theory 15 (1998), 201-252.

[6] Horn, R. and Johnson, C. Matrix analysis, Cambridge university press (2012).

[7] Jackowski, S., Families of subgroups and completion, Journal of Pure and Applied Algebra 37 (1985), $167-179$.

[8] Leary, I. J., A metric Kan-Thurston theorem, Journal of Topology 6(1) (2013), 251-284.

[9] Lück, W., Survey on classifying spaces for families of subgroups, Infinite Groups: Geometric, Combinatorial and Dynamical Aspects, Springer (2005), 269-322.

[10] Lück, W, The Burnside ring and equivariant stable cohomotopy for infinite groups, Journal of Pure and Applied Mathematics Quaterly 1(3) (2005), 479-541. 
[11] Lück, W, Equivariant cohomological Chern characters, International Journal of Algebra and Computation 15 (2005), 1025-1052.

[12] Lück, W, and Oliver, B., The completion theorem in K-theory for proper actions of a discrete group, Topology 40 (2001), 585-616.

[13] Lück, W, and Oliver, B., Chern characters for the equivariant K-theory of proper G-CW-complexes, Cohomological methods in homotopy theory (2001), 217-247.

[14] Lück, W, and Uribe, B., Equivariant principal bundles and their classifying spaces, Algebraic and Geometric Topology 14 (2014), 1925-1995.

[15] May, J.P., Equivariant homotopy and cohomology theory, CBMS regional conferences series in mathematics 91.

[16] Munkres, J. R., Elements of Algebraic Topology, Addison-Wesley (1984).

[17] Sánchez-García, R., Equivariant K-homology for some Coxeter groups, Journal of the London Mathematical Society, 75(3), (2007), 773-790.

[18] Segal, G., Equivariant K-theory, Publ. IHES 34 (1968) 129-151.

[19] Serre, J.-P., Linear representations of finite groups, Graduate Text in Mathematics, Springer, Vol 42 (2012).

[20] t. Dieck, T., Transformation groups, Studies in Math. 8, de Gruyter (1987). 